Article

\title{
Multi-Sensor Building Fire Alarm System with Information Fusion Technology Based on D-S Evidence Theory
}

\section{Qian Ding, Zhenghong Peng, Tianzhen Liu * and Qiaohui Tong}

School of Urban Design, Wuhan University, Bayi Road 16, Wuhan 430072, Hubei, China;

E-Mails: shelleydq@163.com (Q.D.); zhenghongp@yeah.net (Z.P.); irenetqh@126.com (Q.T.)

* Author to whom correspondence should be addressed; E-Mail: tzliu@whu.edu.cn;

Tel.: +86-135-544-307-18; Fax: +86-27-687-720-48.

External Editor: Chen-Chung Liu

Received: 15 June 2014; in revised form: 18 September 2014 / Accepted: 28 September 2014 /

Published: 14 October 2014

\begin{abstract}
Multi-sensor and information fusion technology based on Dempster-Shafer evidence theory is applied in the system of a building fire alarm to realize early detecting and alarming. By using a multi-sensor to monitor the parameters of the fire process, such as light, smoke, temperature, gas and moisture, the range of fire monitoring in space and time is expanded compared with a single-sensor system. Then, the D-S evidence theory is applied to fuse the information from the multi-sensor with the specific fire model, and the fire alarm is more accurate and timely. The proposed method can avoid the failure of the monitoring data effectively, deal with the conflicting evidence from the multi-sensor robustly and improve the reliability of fire warning significantly.
\end{abstract}

Keywords: multi-sensor; information fusion; building; fire alarm; D-S theory

\section{Introduction}

With the rapid development of the economy, the quantity and complexity of buildings have increased, leading to increasing fire hazards. Therefore, fire alarms have become a major issue for many modern buildings. In many buildings, fire alarm systems are equipped with wired or wireless sensors as part of an overall building management system [1]. 
A building fire alarm system consists of sensors, data acquisition and processing modules. Fire detection sensors are the automatic components of the fire alarm system, including sensors for light, smoke, temperature, gas and humidity [2]. They are able to promptly detect the physical and chemical information generated by a fire, such as the light (flame), smoke (smoke particles), heat (temperature), gas (combustion gas) and moisture (air humidity). They can also accurately transmit the physical or chemical information into an electric signal, then rapidly send the signal to the fire alarm controller. This is the process of data collection. There are two kinds of collectors of building fire information: a single-sensor detector and a multi-sensor detector [3-5].

A single-sensor fire detector is vulnerable to interference, such as dust, electromagnetism, water vapor, air, light, vibration and other environmental conditions. It cannot effectively distinguish between the early fire signals and environmental interference signals, so it cannot send early fire warnings correctly. According to statistics from the 1990s, smoke detectors in the United States had an $85 \%$ false alarm rate. In Switzerland, the correct alarm rate (true positives) of single-sensor detectors increased from $9 \%$ in 1995 to $15 \%$ in 2000 [6].

Fire alarm systems currently use three or more sensors to improve the reliability of the system, in order to reduce the false negatives of the fire alarm [7]. When fire information coming from multi-sensor detectors is transmitted into the data center of the processing system, how to deal with the information becomes a major problem. A simple method is to use the maximum value of information to realize the warning. That is, when the observation values of one or more sensors exceed the threshold value, the warning system will be triggered. However, this mode increases the false alarm rate and greatly reduces credibility. In many cases, fire occurs when all of the observation values coming from the multiple sensors do not exceed the threshold value. How to fuse the information collected by a multi-sensor is an urgent problem of building fire alarm technology.

Information fusion technology offers an effective method to combine multi-sensor information. In fact, the fusion process of multi-sensor information is a simulation of the integrated human brain functions for complex problems [8,9]. The basic principle of multi-sensor fusion technology is to utilize the different and complementary sensors in a reasonable manner to make a reliable judgment. In the process of information fusion, certain algorithms and optimization criteria are needed.

Combined with multi-sensor and information fusion technology, a multi-sensor intelligent fire monitoring system (MIFMS) can identify the type of fire accurately and improve the correct fire alarm rate. In Switzerland, the spread of MIFMS from 1995 to 1998 increased the correct alarm rate to 50\%.

The application of multi-sensor information fusion technology to a building fire alarm is mainly via an information fusion algorithm [10]. The algorithms of information fusion technology are predominantly the Bayesian information fusion method, D-S evidence theory, neural networks or fuzzy set theory methods. D-S evidence theory has been widely applied in many research areas. The basic idea of D-S evidence theory was firstly proposed by Dempster when he was studying statistical problems, which was then extended to a more general rule by Shafer. The D-S evidence theory has three main features. The first one is a condition more weakly satisfied than Bayes probability theory, that is to say, additive probability is not necessary. The second is that it can directly express the conditions "uncertain" and "do not know". This information is indicated in a mass function and retained in evidence synthesis. The last feature is that evidence theory not only allows credibility to be given to the individual elements in a space, but also to its subsets. D-S evidence theory has a strong 
theoretical foundation, which not only deals with the uncertainty caused by randomness factors, but also deals with the ambiguity caused by an uncertain environment. It does not require any a priori probability and conditional probability density and has been widely applied in many fields, such as failure diagnosis and pattern recognition [11-15].

This paper introduces multi-sensor data fusion technology based on D-S evidence theory to the field of building fire alarms. The information fusion process includes two dimensions: the time domain and the space domain. Moreover, a fire is a continuous process involving changes in light, smoke, temperature, gas and humidity. These changes are gradually generated, but not synchronously. The integration of time and space is more complex, with more modes in the information fusion technology, which can significantly improve the recognition rate of early fire warning.

This paper is organized as follows. In Section 2, the application of D-S evidence theory for MIFMS is investigated and the system structure is described. Then, the basic properties of D-S theory are discussed, and the calculation process is presented. In Section 3, the equipment and environment settings are covered. Based on this, the proposed method is verified by experimental results. Finally, the conclusion of the paper is drawn in Section 4.

\section{Information Fusion Process Based on D-S Evidence Theory}

In an information fusion system with a multi-sensor, a fire alarm is raised by a combination of data detected from different sensors. The system collects information from sensors, which constitutes the evidence. Then, the system needs to make a judgment by the combination of the evidence, based on $\mathrm{D}-\mathrm{S}$ evidence theory. The principle of D-S theory is to combine different basic probability assignments into a general basic probability assignment with Dempster-Shafer combination rules in a suitable framework. The general basic probability assignment is new evidence. Finally, the system makes a final decision according to the new evidence based on decision rules.

\subsection{Application Principle of D-S Evidence Theory for MIFMS}

In MIFMS, each sensor receives specific information. This information needs to be transmitted to the microprocessor for fusion. Then, the microprocessor makes a decision in cooperation with a central control system. In the end, the system makes a final decision according to the results from subsystems. Therefore, it is appropriate to use the D-S evidence theory as a convergence algorithm. Each subsystem has five sensors. Figure 1 shows the system structure of a MIFMS in which the D-S method is applied. The whole system works as an information fusion process.

In Figure $1, M_{1}\left(A_{i}\right), \ldots, M_{5}\left(A_{i}\right), i=1,2, \ldots, n$ are basic probability assignments to proposition $A_{i}$ from five sensors. $M_{j}\left(A_{i}\right), j=1, \ldots, 5$ are the combined basic probability assignments according to Dempster-Shafer rules. The set of all fire cases is denoted $\Theta$ and is called the alarm framework. Every subset of $\Theta$ is independent. 
Figure 1. Basic system structure of a multi-sensor intelligent fire monitoring system (MIFMS) based on D-S theory.

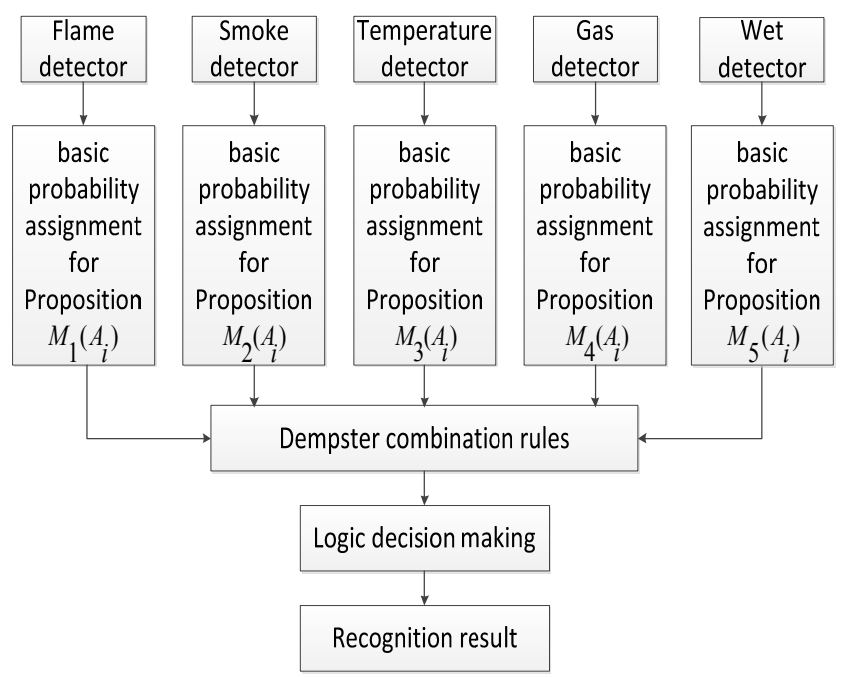

\subsection{Calculation Process for MIFMS}

The basic definitions of D-S evidence theory are described as follows.

(1) Basic probability assignment function: $A_{i}$ is a grade of the information detected by a sensor in alarm framework $\Theta$, if function $M_{j}$ satisfies $M_{j}(\varnothing)=0$ and $\sum_{i} M_{j}\left(A_{i}\right)=1$ under the map $2^{\Theta} \rightarrow[0,1] ; M_{j}$ is called a basic probability assignment function based on $2^{\Theta} . M_{j}\left(A_{i}\right)$ is the basic probability assignment for proposition $A_{i}$ and denotes the degree of trust in the assignment. $A_{i}$ is called the focus element.

(2) Belief function: The belief function denotes the total degree to which a grading of the information is supported by the obtained evidence. For Grades $\mathrm{A}$ and $\mathrm{B}$ satisfying $B \subseteq A$, $A \subseteq \Theta, B \subseteq \Theta$. Define the following function,

$$
\begin{aligned}
& B e l: 2^{\Theta} \rightarrow[0,1] \\
& \operatorname{Bel}(A)=\sum_{B \subseteq A} M(B)
\end{aligned}
$$

$\mathrm{Bel}$ is the belief function of $\Theta$.

(3) Likelihood function: The likelihood function denotes the degree to which the grading cannot be rejected by the obtained evidence. Given a map $P l: 2^{\Theta} \rightarrow[0,1]$, it is defined as:

$$
P l(A)=1-\operatorname{Bel}(\bar{A})=\sum_{B \cap A \neq \phi} M(B)
$$

Equation (3) can be called the likelihood function or upper limit function.

(4) Dempster-Shafer combination rules: Let us consider the belief functions of the first two sensors $B e l_{1}$ and $B_{e l} l_{2} M_{1}$ and $M_{2}$ are the corresponding basic probability assignment functions. The focus elements are, respectively, $A_{1}, A_{2}, \ldots, A_{n}$ and $B_{1}, B_{2}, \ldots, B_{m}$. Then, the new probability $M(C), C=A_{i} \cap B_{j}$ can be derived by combining these two pieces of evidence according to Dempster-Shafer combination rules in the following way. 


$$
M(C)=\left\{\begin{array}{l}
0, C=\phi \\
\frac{1}{1-K} \sum_{A_{i} \cap B_{j}=C} M_{1}\left(A_{i}\right) M_{2}\left(B_{j}\right), C \neq \phi
\end{array}\right\}
$$

Where $K=\sum_{A_{i} \cap B_{j}=\phi} M_{1}\left(A_{i}\right) M_{2}\left(B_{j}\right)$. The rule gives the probability of the cross set of two original sets. The coefficient $K$ guarantees $M(C) \leq 1$.

In Equation (4), if $K \neq 1, M$ makes a certain basic probability assignment. On the other hand, if $K=1$, we regard that $M_{1}$ and $M_{2}$ are contradictory, and it is not possible to combine the two basic probability assignments. For multiple pieces of evidence, we can combine the pieces of evidence one by one. Each subsystem of MIFMS has five pieces of evidence; they are combined step by step.

There are five basic properties of D-S evidence theory.

a. Commutability: $m_{1} \oplus m_{2}=m_{2} \oplus m_{1}$

b. Associability: $m_{1} \oplus\left(m_{2} \oplus m_{3}\right)=\left(m_{1} \oplus m_{2}\right) \oplus m_{3}$

c. Monotonic: Assume that $m_{1}$ and $m_{2}$ are monotonous, when applying the rules to generate a new synthetic $m_{3} ; m_{3}$ is also monotonous.

d. Identity: Let the basic distribution function $m_{0}: 2^{\Theta} \rightarrow[0,1]$ be empty; there is, $m_{0}(\Theta)=1$, $m$ (other) $=0$; for all of the basic probability functions $m$, we have $m \oplus m_{0}=m ; m_{0}$ is the only basic probability assignment (BPA) satisfying the formula.

e. Polarization resistance: The evidence theory of polarization means that for one proposition, when there is an expert opinion, more persuasive synthetic effects are generated than just the one.

(5) Final decision of the microprocessor: The number of fire styles is confirmed by the following method. First, in order to reduce the information amount and lower the burden on the system as much as possible, each signal (light, smoke, temperature, gas and moisture) is only classified into six grades. Thus, the number of fire cases is $6^{5}=7776$ at most. The system will work more efficiently when dealing with less than 7776 species; however, here, 7776 conditions will be discussed. Because there are many fire cases, one microprocessor is set at each detected spot. In this way, each subsystem is an information fusion system. The following is the decision-making process of the microprocessor. The 7776 species constitute a fire alarm framework $\Theta=\left\{H_{1}, H_{2}, \ldots, H_{7776}\right\}$. According to D-S combination rules, the reports are integrated from sensors that support the same supposition. Suppose there are $S_{j}$ reports from five sensors supporting $H_{j}, j=1,2, \ldots, 7776$. Then, the support degree after integrating $S_{j}$ reports is:

$$
\begin{gathered}
M^{S_{j}}\left(H_{j}\right)=1-\prod_{i=1}^{S_{j}} M_{i}(\Theta)=1-\prod_{i=1}^{S_{j}}\left[1-M_{i}\left(H_{j}\right)\right] \\
M^{S_{j}}(\Theta)=\prod_{i=1}^{S_{j}}\left[1-M_{i}\left(H_{j}\right)\right]
\end{gathered}
$$

Where $M^{S_{j}}\left(H_{j}\right)$ is the general degree to which $S_{j}$ sensors support $H_{j}$. 
By D-S combination rules, the denominators of the support degree are the same and can be ignored. Suppose $W$ is the total number of support degrees. $Q^{S_{i}}\left(H_{i}\right)$ is the numerator of the support degrees to which integrated $W$ support $H_{i}$, then:

$$
\begin{aligned}
& Q^{S_{i}}\left(H_{i}\right)-Q^{S_{i}}\left(H_{j}\right)=\frac{M^{S_{i}}\left(H_{i}\right)}{M^{S_{i}}(\Theta)} \prod_{t=1}^{W} M^{S_{t}}(\Theta)-\frac{M^{S_{j}}\left(H_{j}\right)}{M^{S_{j}}(\Theta)} \prod_{t=1}^{W} M^{S_{t}}(\Theta) \\
& =\left[M^{S_{i}}\left(H_{i}\right) M^{S_{j}}(\Theta)-M^{S_{i}}(\Theta) M^{S_{j}}\left(H_{j}\right)\right] \times \prod_{\substack{t=1 \\
t \neq i, j}}^{W} M^{S_{t}}(\Theta) \\
& =\left\{M^{S_{i}}\left(H_{i}\right)\left[1-M^{S_{j}}\left(H_{j}\right)\right]-\left[1-M^{S_{i}}\left(H_{i}\right)\right] M^{S_{j}}\left(H_{j}\right)\right\} \times \prod_{\substack{t=1 \\
t \neq i, j}}^{W} M^{S_{t}}(\Theta) \\
& =\left\{\left[M^{S_{i}}\left(H_{i}\right)-M^{S_{j}}\left(H_{j}\right)\right] \times \prod_{\substack{t=1 \\
t \neq i, j}}^{W} M^{S_{t}}(\Theta)\right. \\
& \left.=\left[\prod_{t=1}^{S_{i}}\left(1-M_{t}\left(H_{i}\right)\right)\right]-\left[1-\prod_{t=1}^{S_{j}}\left(1-M_{t}\left(H_{j}\right)\right)\right]\right\} \times \prod_{\substack{t=1 \\
t \neq i, j}}^{W} M^{S_{t}}(\Theta) \\
&
\end{aligned}
$$

Suppose the above polynomial has a value greater than zero, then:

$$
\prod_{t=1}^{S_{j}}\left(1-M_{t}\left(H_{j}\right)\right)>\prod_{t=1}^{S_{j}}\left(1-M_{t}\left(H_{i}\right)\right)
$$

Taking the logarithm for both sides,

$$
\sum_{t=1}^{S_{j}} \ln \left(1-M_{t}\left(H_{j}\right)\right)-\sum_{t=1}^{S_{j}} \ln \left(1-M_{t}\left(H_{i}\right)\right)>0
$$

The above equation can be written in simple form as $\sum \alpha_{t} \mu_{t}>0$, where:

$$
\alpha_{t}=\left\{\begin{array}{l}
(1 / j) \times \ln \left[1-M_{t}\left(H_{j}\right), \text { when } \mu_{t}=j\right] \\
(-1 / j) \times \ln \left[1-M_{t}\left(H_{i}\right), \text { when } \mu_{t}=i\right]
\end{array}\right\}
$$

In summary, we can get the decision-making rule in the following,

$$
f\left(S_{i}, S_{j}\right)=\left\{\begin{array}{l}
H_{i}, \text { if } \sum \alpha_{t} \mu_{t}>0 \\
H_{j}, \text { otherwise }
\end{array}\right\}
$$

using the above method repeatedly, the microprocessor makes a decision as to whether the fire alarm grade is $H_{m}$. It satisfies $f\left(S_{m}, S_{i}\right)=H_{m}, i=1,2, \ldots, 7776$.

So far, the information fusion process in the space domain has been calculated. However, in the fire detection system, the sensor is periodically or continuously monitoring the target. Fire, in terms of the measurement of light, smoke, temperature, gas and moisture, should be put in sequence, noting that the values are also gradually changing. Some fires start with smoke, then flames appear; others start with flames, and smoke appears only later. Therefore, during the fire detection, for multi-sensor 
measurement data using D-S evidence theory, the analysis of information should be carried out simultaneously in the space and time domain fusion, as shown in Figure 2.

Figure 2. Space-time fusion based on D-S evidence theory.

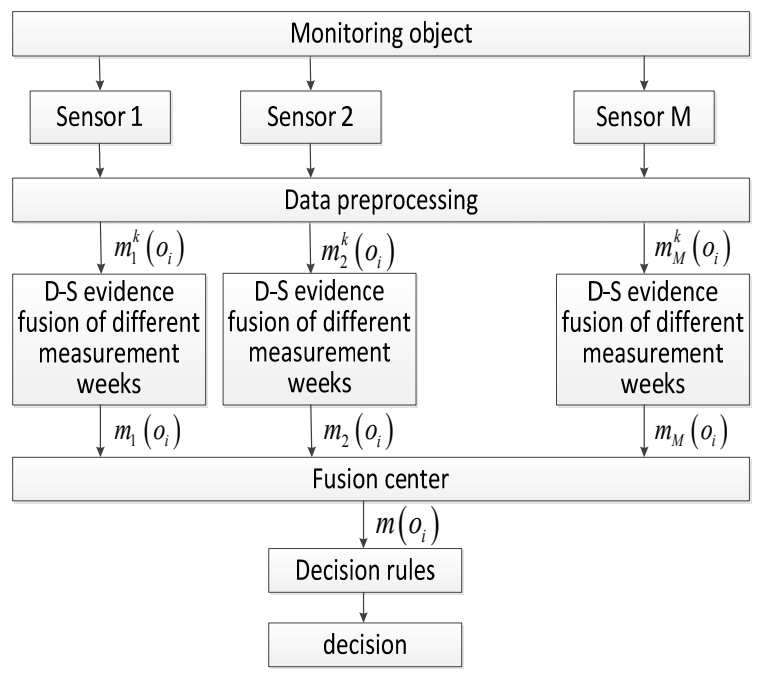

Suppose there are $M$ sensors to detect the scene (in the same recognition framework $\Theta$ ); $m_{s}^{k}\left(o_{i}\right)$ is the $s$-th sensor in the $k$-th measurement cycle calculating the basic probability assignment of the target pattern; here, $s=1,2, \mathrm{~L} . . \mathrm{M}, k=1,2, \mathrm{~L} . . \mathrm{M} . m_{s}(o)$ is the sum of basic probability of mode $o$ from the $s$-th sensor in the $N$-th measurement cycle after fusion of the target.

The calculation process is as follows:

(1) The time domain information fusion should be carried out for each sensor:

$$
m_{s}\left(o_{i}\right)=\frac{\sum_{o_{i} \cap o_{j}=o} \prod_{1 \leq k \leq N} m_{s}^{k}\left(o_{i}\right)}{1-k}
$$

(2) The time domain information fusion of each sensor should update the fusion results for space and time.

$$
m\left(o_{i}\right)=\frac{\sum_{o_{i} \cap o_{j}=o} \prod_{1 \leq s \leq N} m_{s}\left(o_{j}\right)}{1-k}
$$

for which $K=\sum_{o_{i} \cap o_{j}=\phi 1 \leq s \leq N} \prod_{s} m_{s}\left(o_{j}\right)$.

\section{Experimental Results}

An experiment to test a building fire detection warning system based on the multi-sensor and information fusion technology was carried out. The experiment was designed to test the real effect of information fusion technology in the building fire warning system.

\subsection{Select Fire Detection Equipment}

$\mathrm{N}$ sensors were placed in the fire detection system to input data from the fire scene. It is important to select the fire parameters. In general, the content of $\mathrm{CO}$ is low in air. When a fire takes place, 
$\mathrm{CO}$ content will greatly increase. Therefore, the content of CO may be used as an important parameter for the combustion phenomenon in the built environment. Because the building space is essentially closed, fire is often accompanied by a rise in temperature, an increase in smoke concentration, the production of flame radiation and a reduction in humidity. Therefore, we can select five fire parameters: the content of $\mathrm{CO}$, the change of air temperature, the change of smoke concentration, the wavelength of the flame radiation and humidity. The detailed descriptions of the sensors are as follows.

(1) CO gas sensor: A chemical gas detector was used as the CO sensor. The chemical gas detector utilizes a high sensitivity semiconductor, with the characteristics of resistance change when the combustible gas adsorption is discovered, to achieve detection purposes. A FIGARO semiconductor gas sensor TGS2600 was selected for this experiment. It can detect low concentrations of pollutant gases, even a few millionths of a mass percent of the content of polluting gases.

(2) Temperature sensor: Thermal resistance temperature detectors sensitively respond to temperature parameters of a point or a segment within the warning area. The temperature sensor was a HUMIREL HM1500, which is a low cost, small, long life, high stability sensor.

(3) Smoke sensor: In this study, a linear infrared beam smoke detector BEAM1224S produced by Honeywell was used. It has properties, including intelligent judgment, automatic and quick location calibration, strong anti-jamming capability and an automatic dust compensation function. Moreover, it is easy to install.

(4) Flame sensor: A UV photosensitive fire detector is sensitive to ultraviolet radiation by flame radiation. The flame detector used in this experiment was Honeywell's C7027A, which is a small observational UV flame detector.

(5) Humidity sensor: Capacitive-type humidity sensors are typically made of a polymer film capacitor. They commonly incorporate polymer materials, such as polystyrene, polyimide, cellulose acetate acid, and so on. In this work, the humidity measurement module AM2301 based on the capacitive-type humidity sensor was used. By using this industrial-grade, high-performance device, factors, such as measurement accuracy, wide temperature range, compactness, ease-of-use, fast response and low power consumption, reached high specifications.

\subsection{Laboratory and Expert Database Installation}

The internal space size of the laboratory used was $8 \mathrm{~m}$ (length) $\times 5 \mathrm{~m}$ (width) $\times 3.6 \mathrm{~m}$ (height), with a camera and vent. There is a glass observation window in a wall. Five sensors: a gas sensor, temperature sensors, an ionization smoke sensor, a flame sensor and a humidity sensor were installed on the ceiling of the laboratory. We use the camera to observe specific combustion conditions and recorded at key points in time.

We have constructed the corresponding expert database as shown in Table 1. In order to prevent exaggerating certain effects, the data entered into the expert database were normalized to the interval $[0,1]$. Moreover, the normalization procedure minimizes the error brought by variations in conditions and precisely represents the probability of fire. 
Table 1. Expert database for a multi-sensor intelligent fire monitoring system (MIFMS).

\begin{tabular}{|c|c|c|c|c|c|c|}
\hline Number & $\mathrm{CO}$ & Temperature & Smoke & Flame & Humidity & Probability of Fire \\
\hline 1 & 0.1 & 0.2 & 0.2 & 0.2 & 0.2 & 0.20 \\
\hline 2 & 0.2 & 0.3 & 0.2 & 0.3 & 0.5 & 0.30 \\
\hline 3 & 0.3 & 0.4 & 0.3 & 0.2 & 0.4 & 0.34 \\
\hline 4 & 0.5 & 0.2 & 0.4 & 0.3 & 0.3 & 0.40 \\
\hline 5 & 0.2 & 0.4 & 0.4 & 0.3 & 0.5 & 0.40 \\
\hline 6 & 0.2 & 0.3 & 0.5 & 0.3 & 0.6 & 0.40 \\
\hline 7 & 0.6 & 0.4 & 0.2 & 0.5 & 0.3 & 0.43 \\
\hline 8 & 0.7 & 0.2 & 0.5 & 0.4 & 0.3 & 0.45 \\
\hline 9 & 0.1 & 0.6 & 0.7 & 0.4 & 0.4 & 0.49 \\
\hline 10 & 0.6 & 0.3 & 0.6 & 0.4 & 0.4 & 0.50 \\
\hline 11 & 0.4 & 0.4 & 0.7 & 0.4 & 0.5 & 0.50 \\
\hline 12 & 0.7 & 0.4 & 0.5 & 0.5 & 0.4 & 0.50 \\
\hline 13 & 0.9 & 0.3 & 0.5 & 0.5 & 0.4 & 0.54 \\
\hline 14 & 0.5 & 0.4 & 0.8 & 0.4 & 0.6 & 0.58 \\
\hline 15 & 0.3 & 0.7 & 0.7 & 0.5 & 0.6 & 0.58 \\
\hline 16 & 0.2 & 0.8 & 0.6 & 0.5 & 0.7 & 0.60 \\
\hline 17 & 0.3 & 0.7 & 0.8 & 0.4 & 0.7 & 0.61 \\
\hline 18 & 0.8 & 0.4 & 0.7 & 0.5 & 0.5 & 0.65 \\
\hline 19 & 0.4 & 0.7 & 0.7 & 0.5 & 0.7 & 0.70 \\
\hline 20 & 0.5 & 0.6 & 0.9 & 0.5 & 0.6 & 0.71 \\
\hline 21 & 0.3 & 0.9 & 0.7 & 0.5 & 0.8 & 0.76 \\
\hline 22 & 0.2 & 0.9 & 0.8 & 0.6 & 0.8 & 0.77 \\
\hline 23 & 0.8 & 0.7 & 0.6 & 0.7 & 0.6 & 0.80 \\
\hline 24 & 0.9 & 0.5 & 0.9 & 0.6 & 0.6 & 0.80 \\
\hline 25 & 0.7 & 0.7 & 0.9 & 0.6 & 0.7 & 0.82 \\
\hline 26 & 0.6 & 0.7 & 1.0 & 0.6 & 0.8 & 0.84 \\
\hline 27 & 0.9 & 0.6 & 0.9 & 0.7 & 0.7 & 0.86 \\
\hline 28 & 0.9 & 0.8 & 0.7 & 0.8 & 0.7 & 0.90 \\
\hline 29 & 0.8 & 0.7 & 0.8 & 0.9 & 0.8 & 0.90 \\
\hline 30 & 0.9 & 0.8 & 0.8 & 0.8 & 0.8 & 1.00 \\
\hline
\end{tabular}

\subsection{Test Ignitions}

Test ignitions were produced by pyrolysis of wood, cotton rope or plastic. We used an electric stove to heat wood, paper, cotton rope and polyurethane material to generate fire.

\subsection{Experiment Steps}

The experiment was carried out according to the following steps.

Step 1: Set the condition of the laboratory. In this step, we chose several different conditions for the test. For example, $\mathrm{CO}$ gas was released into the room when there was no fire.

Step 2: Monitoring the data collected by the five sensors. The central processor read the data of the sensors when the environmental conditions were stable in the laboratory. 
Step 3: Normalize the monitoring data. Since the data collected by the sensors are physical variables, they were normalized to the probability of fire according to the expert database.

Step 4: Use D-S theory for the fire alarm decision.

\subsection{Experiment Results}

\subsubsection{Condition 1: no fire}

The first experiment was carried out when there was no fire or other abnormal conditions in the laboratory. The real-time monitoring data were collected, then normalized as the fire probability, shown as Table 2.

As Table 2 shows, all of the evidence collected by the five sensors was normalized. The flame sensor and humidity sensor show clearly that there is no fire. The temperature in the laboratory is relatively high, so the probability of fire is 0.1930 . However, taken together, the evidence data tend towards the conclusion that there is no fire, and the fusion result coincided with this conclusion.

Table 2. Fire probability data and fusion result under Condition 1.

\begin{tabular}{ccc}
\hline \multirow{2}{*}{ Probability } & \multicolumn{2}{c}{ Evidence 1 } \\
\cline { 2 - 3 } & Fire & No Fire \\
\hline Temperature & 0.1930 & 0.8070 \\
Smoke & 0.1532 & 0.8468 \\
CO & 0.0225 & 0.9775 \\
Flame & 0.0000 & 1.0000 \\
Humidity & 0.0000 & 1.0000 \\
Fusion result & 0.0258 & 0.9742 \\
\hline Real condition & \multicolumn{2}{c}{ No fire } \\
\hline
\end{tabular}

\subsubsection{Condition 2: fire}

The second experiment was under the condition that there is fire in the laboratory. The real-time monitoring data were collected and normalized to the fire probability, as shown as Table 3.

Table 3. Fire probability data and fusion result under Condition 2.

\begin{tabular}{ccc}
\hline \multirow{2}{*}{ Probability } & \multicolumn{2}{c}{ Evidence 2 } \\
\cline { 2 - 3 } & Fire & No Fire \\
\hline Temperature & 0.8014 & 0.1986 \\
Smoke & 0.5277 & 0.4723 \\
CO & 0.9270 & 0.0730 \\
Flame & 0.5174 & 0.4826 \\
Humidity & 0.8972 & 0.1028 \\
Fusion result & 0.7258 & 0.2742 \\
\hline Real condition & \multicolumn{2}{c}{ Fire } \\
\hline
\end{tabular}

Since all of the evidence shows that the probability of fire is higher than the probability of no fire, the fusion result is that the probability of fire is 0.7258 . 


\subsubsection{Condition 3: smoke released}

In the third experiment, smoke was released into the laboratory by using the smoke tester, HZJC-1, while the other conditions were the same as Condition 1. The real-time monitoring data are transferred to the fire probability, shown as Table 4 .

Table 4. Fire probability data and fusion result in Condition 3.

\begin{tabular}{ccc}
\hline \multirow{2}{*}{ Probability } & \multicolumn{2}{c}{ Evidence 3 } \\
\cline { 2 - 3 } & Fire & No Fire \\
\hline Temperature & 0.1866 & 0.8134 \\
Smoke & 0.8742 & 0.1258 \\
CO & 0.3911 & 0.6089 \\
Flame & 0.0800 & 0.9200 \\
Humidity & 0.1264 & 0.8736 \\
Fusion result & 0.1532 & 0.8468 \\
\hline Real condition & \multicolumn{2}{c}{ No fire } \\
\hline
\end{tabular}

The smoke evidence shows that the probability of fire is high, which is different from the evidence collected by other sensors. However, by using the D-S evidence theory, the final fusion results can effectively avoid the disturbance brought by the smoke.

\subsubsection{Condition 4: smoldering condition}

A large number of paper rolls were closely arranged and placed on a heating plate with a power of $2 \mathrm{~kW}$. After the heating process, smoldering was produced.

From Table 5, CO evidence shows that the probability of fire is high, while the evidence collected by other sensors shows that the fire probability is at a relatively low level. Using the D-S evidence theory, the final fusion results concluded that there is a fire.

Table 5. Fire probability data and fusion result under Condition 3.

\begin{tabular}{ccc}
\hline \multirow{2}{*}{ Probability } & \multicolumn{2}{c}{ Evidence 1 } \\
\cline { 2 - 3 } & Fire & No Fire \\
\hline Temperature & 0.5448 & 0.4552 \\
Smoke & 0.5275 & 0.4725 \\
CO & 0.9211 & 0.0789 \\
Flame & 0.1250 & 0.8750 \\
Humidity & 0.3369 & 0.6631 \\
Fusion result & 0.6037 & 0.3963 \\
\hline Real condition & \multicolumn{2}{c}{ Fire } \\
\hline
\end{tabular}

\subsubsection{Experiment with the improved expert database}

The expert database can be improved by adding an uncertainty probability, as shown in Table 6 . The last column of Table 6 is the uncertainty probability, which represents that it is hard to judge whether there is fire according to the current data collected by the sensors. The uncertainty probability 
decreases when the probability of fire increases, which is more reasonable than Table 1. On the basis of Table 6, the fusion result should also have three terms: the probability of fire, the probability of no fire and the uncertainty probability.

Table 6. Improved expert database for MIFMS.

\begin{tabular}{|c|c|c|c|c|c|c|c|c|}
\hline Number & $\mathrm{CO}$ & Temperature & Smoke & Flame & Humidity & $\begin{array}{c}\text { Probability } \\
\text { of Fire }\end{array}$ & $\begin{array}{l}\text { Probability } \\
\text { of No Fire }\end{array}$ & $\begin{array}{l}\text { Uncertainty } \\
\text { Probability }\end{array}$ \\
\hline 1 & 0.1 & 0.2 & 0.2 & 0.2 & 0.2 & 0.20 & 0.65 & 0.10 \\
\hline 2 & 0.2 & 0.3 & 0.2 & 0.3 & 0.5 & 0.30 & 0.60 & 0.10 \\
\hline 3 & 0.3 & 0.4 & 0.3 & 0.2 & 0.4 & 0.34 & 0.58 & 0.10 \\
\hline 4 & 0.5 & 0.2 & 0.4 & 0.3 & 0.3 & 0.40 & 0.50 & 0.10 \\
\hline 5 & 0.2 & 0.4 & 0.4 & 0.3 & 0.5 & 0.40 & 0.47 & 0.10 \\
\hline 6 & 0.2 & 0.3 & 0.5 & 0.3 & 0.6 & 0.40 & 0.45 & 0.10 \\
\hline 7 & 0.6 & 0.4 & 0.2 & 0.5 & 0.3 & 0.43 & 0.43 & 0.10 \\
\hline 8 & 0.7 & 0.2 & 0.5 & 0.4 & 0.3 & 0.45 & 0.41 & 0.10 \\
\hline 9 & 0.1 & 0.6 & 0.7 & 0.4 & 0.4 & 0.49 & 0.40 & 0.10 \\
\hline 10 & 0.6 & 0.3 & 0.6 & 0.4 & 0.4 & 0.50 & 0.40 & 0.10 \\
\hline 11 & 0.4 & 0.4 & 0.7 & 0.4 & 0.5 & 0.50 & 0.40 & 0.09 \\
\hline 12 & 0.7 & 0.4 & 0.5 & 0.5 & 0.4 & 0.50 & 0.34 & 0.09 \\
\hline 13 & 0.9 & 0.3 & 0.5 & 0.5 & 0.4 & 0.54 & 0.32 & 0.09 \\
\hline 14 & 0.5 & 0.4 & 0.8 & 0.4 & 0.6 & 0.58 & 0.32 & 0.09 \\
\hline 15 & 0.3 & 0.7 & 0.7 & 0.5 & 0.6 & 0.58 & 0.31 & 0.09 \\
\hline 16 & 0.2 & 0.8 & 0.6 & 0.5 & 0.7 & 0.60 & 0.27 & 0.09 \\
\hline 17 & 0.3 & 0.7 & 0.8 & 0.4 & 0.7 & 0.61 & 0.26 & 0.09 \\
\hline 18 & 0.8 & 0.4 & 0.7 & 0.5 & 0.5 & 0.65 & 0.22 & 0.08 \\
\hline 19 & 0.4 & 0.7 & 0.7 & 0.5 & 0.7 & 0.70 & 0.20 & 0.08 \\
\hline 20 & 0.5 & 0.6 & 0.9 & 0.5 & 0.6 & 0.71 & 0.18 & 0.07 \\
\hline 21 & 0.3 & 0.9 & 0.7 & 0.5 & 0.8 & 0.76 & 0.16 & 0.06 \\
\hline 22 & 0.2 & 0.9 & 0.8 & $\mathrm{~s}$ & 0.8 & 0.77 & 0.15 & 0.05 \\
\hline 23 & 0.8 & 0.7 & 0.6 & 0.7 & 0.6 & 0.80 & 0.14 & 0.04 \\
\hline 24 & 0.9 & 0.5 & 0.9 & 0.6 & 0.6 & 0.80 & 0.14 & 0.04 \\
\hline 25 & 0.7 & 0.7 & 0.9 & 0.6 & 0.7 & 0.82 & 0.12 & 0.02 \\
\hline 26 & 0.6 & 0.7 & 1.0 & 0.6 & 0.8 & 0.84 & 0.10 & 0.01 \\
\hline 27 & 0.9 & 0.6 & 0.9 & 0.7 & 0.7 & 0.86 & 0.09 & 0.01 \\
\hline 28 & 0.9 & 0.8 & 0.7 & 0.8 & 0.7 & 0.90 & 0.09 & 0.01 \\
\hline 29 & 0.8 & 0.7 & 0.8 & 0.9 & 0.8 & 0.90 & 0.04 & 0.00 \\
\hline 30 & 0.9 & 0.8 & 0.8 & 0.8 & 0.8 & 1.00 & 0.00 & 0.00 \\
\hline
\end{tabular}

The alarm framework is defined as $\Theta=\{Y, N, \ldots, U\} ; Y$ denotes that a fire has taken place; $N$ denotes without fire; and $U$ denotes uncertainty about whether there is a fire. We used an automatic generator to heat materials for $1 \mathrm{~min}$, then used the five sensors to obtain the data for three periods of time, where each period lasted $30 \mathrm{~s}$. We give some basic probability values for the five sensors, based on specialist knowledge and the basic probability values of all sensors in Table 7 . 
Table 7. Basic probability values of all sensors during three time periods.

\begin{tabular}{cccc}
\hline $\boldsymbol{m}_{\boldsymbol{s} j}$ & $Y$ & $N$ & $U$ \\
\hline$m_{11}$ & 0.40 & 0.3 & 0.30 \\
$m_{12}$ & 0.52 & 0.23 & 0.25 \\
$m_{13}$ & 0.77 & 0.18 & 0.05 \\
$m_{21}$ & 0.65 & 0.2 & 0.15 \\
$m_{22}$ & 0.71 & 0.19 & 0.10 \\
$m_{23}$ & 0.85 & 0.13 & 0.02 \\
$m_{31}$ & 0.46 & 0.28 & 0.26 \\
$m_{32}$ & 0.62 & 0.18 & 0.20 \\
$m_{33}$ & 0.82 & 0.14 & 0.04 \\
$m_{41}$ & 0.58 & 0.19 & 0.23 \\
$m_{42}$ & 0.71 & 0.21 & 0.08 \\
$m_{43}$ & 0.91 & 0.08 & 0.01 \\
$m_{51}$ & 0.30 & 0.37 & 0.33 \\
$m_{52}$ & 0.45 & 0.29 & 0.26 \\
$m_{53}$ & 0.63 & 0.18 & 0.19 \\
\hline
\end{tabular}

$m_{s j}$ means the $s$ th sensor in the $j$ th cycle to determine basic probability values.

The basic probability values of given sensors are fused with three periods in the time domain by Formula (12), and the results are shown in Table 8.

Table 8. The accumulation of basic probability values by five sensors with three periods.

\begin{tabular}{cccc}
\hline $\boldsymbol{m}_{\boldsymbol{s} j}$ & $Y$ & $N$ & $U$ \\
\hline$m_{1}$ & 0.6548 & 0.2836 & 0.0978 \\
$m_{2}$ & 0.8237 & 0.1322 & 0.0365 \\
$m_{3}$ & 0.4627 & 0.4786 & 0.1087 \\
$m_{4}$ & 0.5215 & 0.5482 & 0.1042 \\
$m_{5}$ & 0.6952 & 0.2830 & 0.0962 \\
\hline
\end{tabular}

$m_{s j}$ means the $s$ th sensor in the $j$ th cycle to determine basic probability values.

Finally, we fuse the accumulation of basic probability values from five sensors with three periods in the space domain via Formula (13), and the results are $m(Y)=0.65, m(N)=0.25, m(U)=0.1$. According to the decision method based on the basic probability value, when the threshold values are $\varepsilon_{1}=\varepsilon_{2}=0.4$, the last decision is $Y$; that is, there is fire.

\section{Conclusions}

Fire is a complex phenomenon, with a variety of characteristic parameters. It is difficult to achieve high accuracy and wide applicability in the detection of the early stages of fire by measuring a single parameter, while sensing multiple parameters can accurately reflect the comprehensive features of a real fire phenomenon. This paper applies the information fusion method for a multi-sensor with different measurements to a fire alarm system.

A construction method using a basic probability assignment function is proposed. The improved D-S synthetic formula can effectively solve the problem of conflicting evidence, integrating smoke, 
temperature, light and other major fire parameters and eliminating redundancies and contradictions that exist in multi-sensor information. Therefore, the proposed method can effectively avoid false positives, underreporting and alarm delays. The calculation results show that the proposed algorithm improves fusion results significantly.

\section{Acknowledgments}

The authors are grateful to the editors and reviewers for their insightful comments and suggestions. This study was supported by the National Natural Science Foundation of China (81271513, 91324201) and the Fundamental Research Funds for the Central Universities (2042014kf0043).

\section{Author Contributions}

The idea for this research work was proposed by Zhenghong Peng and Qiaohui Tong, and the paper writing was completed by Tianzhen Liu and Qian Ding.

\section{Conflicts of Interest}

The authors declare no conflict of interest.

\section{References}

1. Zeng, Y.; Sreenan, C.J.; Sitanayah, L.; Xiong, N.; Park, J.H.; Zheng, G. An emergency-adaptive routing scheme for wireless sensor networks for building fire hazard monitoring. Sensors 2011, 11, 2899-2919.

2. Beaubrun, R.; Kraimia, Y. Experiments for fire detection using a wireless sensor network. In Proceedings of the Sixth International Conference on Sensor Technologies and Applications, Rome, Italy, 19-24 August 2012; pp. 312-316.

3. $\mathrm{Hu}, \mathrm{X}$. Application of fuzzy data fusion in multi-sensor fire monitoring. In Proceedings of the 2012 International Symposium on Instrumentation \& Measurement, Sensor Network and Automation (IMSNA), Sanya, China, 25-28 August 2012; pp. 157-159.

4. Durrant-Whyte, H.F. Sensor models and multi-sensor integration. Int. J. Robot. Res. 1988, 17, 97-113.

5. Bogler, P.L. Shafer-dempster reasoning with applications to multi-sensor target identification systems. Syst. Man Cybern. 1987, 17, 968-977.

6. Aleksic, Z.J. Minimization of the optical smoke detector false alarm probability by optimizing its frequency characteristic. Instrum. Meas. 2000, 49, 37-42.

7. Preece, P.D.A.; Chen, F.; Porta, T.L.; Bar-Noy, A. A distributed architecture for heterogeneous multi sensor-task allocation. In Proceedings of the 2011 International Conference on Distributed Computing in Sensor Systems and Workshops (DCOSS), Barcelona, Spain, 27-29 June 2011; pp. 1-8.

8. Lia, C.; Heinemannb, H.; Sherryc, P.R. Neural network and Bayesian network fusion models to fuse electronic nose and surface acoustic wave sensor data for apple defect detection. Sens. Actuators B Chem. 2007, 125, 301-310. 
9. Pearson, J.C.; Gelfand, J.J.; Peterson, R.M.; Spence, C.D.; Sullivan, W.E. Neural network approach to sensory fusion. SPIE Sens. Fusion. 1988, 931, 103-108.

10. Zhang, Y.H.; Xu, J.; Du, K. Information fusion algorithm for electromechanical equipment based on DS evidence theory. Appl. Mech. Mater. 2013, 8, 1125-1128.

11. Sung, W.T.; Chang, K.Y. Evidence-based multi-sensor information fusion for remote health care systems. Sens. Actuators A Phys. 2013, 204, 1-19.

12. Feng, R.; Che, S.; Wang, X.; Yu, N. Trust management scheme Based on D-S evidence theory for wireless sensor networks. Int. J. Distrib. Sens. Netw. 2013, 2013, doi:10.1155/2013/948641.

13. Yang, J.; Madah, G.S. An evidential reasoning approach for multiple-attribute decision making with uncertainty. Syst. Man Cybern. 1994, 24, 1-17.

14. Lou, R.C.; Kay, M.G. Data fusion and sensor integration: State of the art 1990s. In Data Fusion in Robotics and Machine Intelligence; Abidi, M.A., Gonzalez, R.C., Eds.; Academic Press Inc.: San Diego, CA, USA, 1992; pp. 127-135.

15. Lin, Y.; Gao, L.; Li, Y.; Si, X. The application of improving space-time D-S evidence theory in distinguishing vehicle. In Proceedings of the Asia Pacific Conference on Postgraduate Research in Microelectronics \& Electronics, Shanghai, China, 19-21 November 2009; pp. 376-379.

(C) 2014 by the authors; licensee MDPI, Basel, Switzerland. This article is an open access article distributed under the terms and conditions of the Creative Commons Attribution license (http://creativecommons.org/licenses/by/4.0/). 